\title{
A Review of Studies on Rogerian Rhetoric and Its Implications for English Writing Instruction in East Asian Countries
}

\author{
Yanling Guo \\ Beijing University of Posts and Telecommunications, China \\ Barry M. Kroll \\ Lehigh University, Pennsylvania, USA
}

\begin{abstract}
This paper first introduces the theoretical source and clarifies the definition of Rogerian rhetoric, then systematically introduces its goals and corresponding strategies, and structures, analyzes the major differences between traditional and Rogerian rhetoric and summarizes the main critiques from three aspects. Finally, it focuses on presenting the related studies in English language teaching in the U.S.A. and summarizing some implications for English writing teaching in East Asian countries.
\end{abstract}

Index Terms-Rogerian rhetoric, empathetic listening, teaching of writing

This paper comprehensively summarizes the studies on Rogerian rhetoric and explores the pedagogical implications for English argument writing teaching in East Asian countries. The authors first introduce the theoretical source of Rogerian rhetoric, which is Rogers' "client-centered" psychotherapy. On the basis of this summary of Rogers' approach, the authors then formulate a tentative definition for Rogerian rhetoric. The paper then summarizes the preliminary, intermediate and final goals of Rogerian rhetoric. Furthermore, the paper introduces Young, Becker and Pike's and Hairston's recommendations for writing a Rogerian argument paper. The authors then analyze the major differences between Rogerian rhetoric and traditional rhetoric in terms of assumption, context, and goal. In the following part, the authors summarize the main critiques of Rogerian rhetoric which mainly center on whether Rogerian rhetoric is truly different from traditional argument; whether Rogerian rhetoric is true to its theoretical source; whether Rogerian rhetoric's requirement of empathetic listening and promotion of neutral language further women's self-effacement; and their corresponding counter arguments. The paper further summarizes the application of Rogerian rhetoric in teaching writing in American institutions of higher education, and explores how Rogerian rhetoric can be implemented in East Asian English writing class to enhance East Asian EFL Learners' writing ability and language skills, broaden their approaches in argument writing, and provide them with flexible choices of argument approaches suitable to diverse contexts.

\section{TheORETICAL SOURCE AND Definition of Rogerian RHETORIC}

\section{A. Theoretical Source}

Rogerian rhetoric, established by Young, Becker and Pike (1970) as an alternative to traditional rhetoric, has drawn its inspiration mainly from Rogers' "client-centered" psychotherapy, which emphasizes empathetic listening. The goal of Rogers' "client-centered" approach is to assist clients in reaching their full potential by creating a supportive and non-evaluative environment, which depends on whether a therapist can meet the following requirements: genuineness or congruence, unconditional positive regard, and empathetic understanding. To Rogers, empathetic understanding means the thorough comprehension of the feelings and meanings of the client and conveying of this "acceptant understanding" back to the client, which, in Rogerian argument is transferred to the understanding of the audience. However, the most direct source for Rogerian rhetoric is Rogers' 1951 paper "Communication: Its Blocking and Its Facilitation," in which Rogers adapts his psychotherapy theory to communication. In it, Rogers (1951) claimed that the major barrier to communication is our "very natural tendency to judge, to evaluate, to approve and disapprove, the statement of the other person, or the other group" (as cited in Young, Becker, \& Pike, 1970, p.28). In the same paper, Rogers also emphasizes that the tendency to evaluate is heightened in emotionally charged situations. To Rogers, the removing of the barrier lies in "listening with understanding", which, according to him, "means to see the expressed idea and attitude from the other person's point of view, to sense how it feels to him, to achieve his frame of reference in regard to the thing he is talking about." These statements become major sources for Rogerian rhetoric.

\section{B. The Definition}

In 1970, Young et al. put forward Rogerian rhetoric in their influential text Rhetoric: Discovery and Change. This new argument approach strives for cooperation and communication rather than coercion in situations where 
commitments and emotions are high. The method attempts to achieve threat-reduction and trust building by first delineating the opponent's views as accurately as possible and searching for shared beliefs and interests, paving the way for cooperation. The issues for which Rogerian rhetoric proves efficacious involve ethical disputes on topics such as race, sexual behavior, personal behavior, and professional standards (Hairston, 1976, p.373). In such situations, as Young et al. (1970) pointed out, "A strong sense of threat may render the reader immune to even the most carefully reasoned and well-supported argument" (p. 274). Therefore, a Rogerian approach is designed to reduce threat, so that the reader will be able to consider alternatives and explore options for eliminating conflict. This is especially important when a conflict is so extreme that two parties can barely listen to one another, to say nothing of rational discussion of their views. As Lamm and Everett pointed out (2007), Rogerian argument is most valuable when a person disagrees "vehemently with another point of view" (p. 283).

However, neither Young et al., nor any other scholars who have been studying and applying Rogerian rhetoric, have provided a clear and concise definition of Rogerian rhetoric. Therefore, the authors formulate the following working definition: Rogerian rhetoric is a method of written argument that's derived from Rogers' client-centered approach to psychotherapy, which prioritizes empathetic listening. The method is an extension of Rogers' claim that listening is central to effective communication, especially when people disagree or are in conflict. In Rogerian rhetoric, a writer expresses the opposing viewpoint in language that is respectful and objective, in order to demonstrate careful listening and lower the sense of threat. The writer identifies points of agreement or connection with the opposition and then shifts to the writer's differing viewpoint, which, if the reader is induced to reciprocate, will get a fair hearing in return.

\section{GOALS AND CORRESPONDING STRATEGIES}

\section{A. The Preliminary Goal and Strategy}

The preliminary goal of Rogerian argument is to reduce threat, which will pave the way for reasonable discussion or genuine communication. To achieve this, Young et al. (1970) proposed to "convey to the reader that he is understood and to delineate the area within which he believes the reader's position to be valid" (p.275). The key lies in "empathy," which, according to Young et al. (1970), " requires getting inside the other person's skin and seeing the world through his eyes, or, to speak less metaphorically, it requires considering the beliefs and perspectives of the reader in the context of his attitudes, values, and past experience" (p.275). The emphasis on "context" is important, because by focusing on contexts in which a position is valid instead of criticizing its legitimacy, the writer's argument has different psychological impact: the former shows the sincerity of the writer's desire to understand, while the later shows the animosity of the writer.

\section{B. The Intermediate Goal and Strategy}

The intermediate goal is to achieve mutual understanding by stating the writer's position. Although this is not the part which makes Rogerian rhetoric unique, how this part is accomplished can either shatter all the efforts of the first phase or finally secure the bridge the writer has been so painstakingly working to build. If the writer illustrates his own position with loaded language, the opponent will have a strong sense of deception, because the opponent believes what the writer has been doing are all tricks or setting traps, which only serve the interests of the writer. Therefore, Young et al. (1970) emphasized that "the writer should avoid evaluative statements whenever description alone is sufficient for his purpose. But when, as often happens, he must make judgments, he can make them in a way that minimizes the reader's sense of threat"' (p. 206).

\section{The Final Goal and Strategy}

The goal for the final phase is either problem-solving or cooperation. Through stating how two positions can be mutually beneficial or complement each other, the writer will probably reach an agreement with the reader on the best-possible solution. Actually, the three goals of the different phases contribute to the ultimate goal, which is "to induce changes in an opponent's mind in order to make mutually advantageous cooperation possible" (Young et al., 1970, p. 283).

\section{The Structures OF Rogerian RHETORIC}

\section{A. Young, Becker and Pike's Structure}

Though there are no "defined structures" to fulfill the goals, Young, Becker and Pike (1970) did provide general phases to organize a Rogerian argument:

(1) An introduction to the problem and a demonstration that the opponent's position is understood.

(2) A statement of the contexts in which the opponent's position may be valid.

(3) A statement of the writer's position, including the contexts in which it is valid.

(4) A statement of how the opponent's position would benefit if he were to adopt elements of the writer's position. If the writer can show that the positions complement each other, that each supplies what the other lacks, so much the better. (p. 283)

\section{B. Hairston's Structure}


Based on Young, Becker and Pike's structure, Hairston (1976) later developed a similar organizational pattern for Rogerian Argument:

(1) Give a brief, objective statement of the issue under discussion.

(2) Summarize in impartial language what you perceive the case for the opposition to be; the summary should demonstrate that you understand their interests and concerns and should avoid any hint of hostility.

(3) Make an objective statement of your own side of the issue, listing your concerns and interests, but avoiding loaded language or any hint of moral superiority.

(4) Outline what common ground or mutual concerns you and the other person or group seem to share; if you see irreconcilable interests, specify what they are.

(5) Outline the solution you propose, pointing out what both sides may gain from it. (p. 210-211)

\section{Miller's Structure}

A textbook written by Miller (2007) echoes this structure, which has now become widely accepted:

(1) Introduction

(2) Summary of Opposing Views

(3) Statement of Your Position

(4) Statement of Contexts

(5) Statement of Benefits (p.132-33)

\section{Traditional Argument And Rogerian Argument}

The study of the relationship between traditional and Rogerian rhetoric, and their differences in particular, has been given ample attention since the birth of the new approach. Actually, Young, Becker and Pike devoted a section in Chapter 12 of their book Rhetoric: Discovery and Change to the analysis of the distinctiveness of Rogerian argument compared to that of the traditional one: they analyzed the differences in areas of logic, language, goal, technique, structure, etc. Following them, more papers were published - primarily in the decade of the 1980s--to further explore these differences (Lunsford, 1979; Bator, 1980; Mader, 1984, Ede, 1984; Coe, 1992). Some of these theorists focus on uniqueness, while others center on criticism. This section will focus on the distinctiveness of Rogerian rhetoric, and the next will give due attention to the criticisms.

\section{A. Difference in Assumptions}

From the perspective of Rogerian theory, classical approaches to rhetoric assume that human beings are reasonable and can be swayed by rational argument (Young et al., 1970, p. 7). Bator (1980), for example, claimed that the "underlying principle of Aristotle's Rhetoric is that man is a rational animal, capable of using logical reasoning as the basis for argument" (p. 427). It is at times true, of course, that humans are rational and can be swayed by logical arguments. Logic does work when we hold homogenous beliefs or values. However, with the development of globalization, people who have diverse religions, beliefs and value systems must learn to communicate despite differences. When disagreement occurs in these situations, argument based on logical coercion probably won't yield desirable results, because it would threaten identity and integrity (Young et al., 1970, p. 7; Bator, 1980, p. 427). Thus, Rogerian rhetoric provides a distinctive contribution to contemporary argument.

\section{B. Difference in Contexts: Triadic vs. Dyadic}

Traditional and Rogerian rhetoric also suit different contexts: traditional argument is designed for the triadic context or advocacy situation, whereas Rogerian argument is based on a dyadic context, or non-advocacy situation. According to Brent (1996), traditional argument is best for triadic context because "it (traditional argument) is aimed at a third party who will judge the case on the basis of the arguments presented by competing advocates, politicians, researchers, advertisers, or other partisan arguers. In this case it matters little if one arguer threatens the beliefs and self-esteem of the other, for it is not the opponent the arguer is trying to convince, but the audience as third party" (p. 77).

In many circumstances, however, two parties are in fact trying to convince each other. These "dyadic" situations are common in everyday life as well as in most deliberative contexts. In these situations, a persuasive approach can often harden the opposition's position. Rogerian rhetoric offers some strategies that will open the opposition to reasonable discussion — which is, ironically, the grounds presupposed by classical argument theory (Coe, 1992; Brent, 1996).

\section{Difference in Goals}

The goals of traditional and Rogerian rhetoric are also drastically different. Young et al. (1970) summarized the difference as follows: "The goal of traditional argument is to make your position prevail .... The goal of Rogerian argument is to create a situation conducive to cooperation" (p. 282). Since the sole purpose of the traditional argument is to win, both sides try to seek "not truth, but advantage" (Coe, 1992, p. 86). Conversely, Rogerian argument, to Thorne (2003), is a process of "discovery" and the goal is "to participate in a dialogue, not to defeat an opponent" (p. 8). This participation may not lead the reader to switch to the writer's viewpoint, but it might result in the invention of a new solution to the problem or combination of the reader's and writer's best ideas. Therefore, it is a "win-win solution" instead of the "zero-sum" situation that typically results in the traditional approach. 


\section{CRitique}

Like any new theory, Rogerian rhetoric has not only attracted appreciation and application, but also critique. The critique can generally be summarized into the following categories: (1) Rogerian argument is not truly different from Aristotelian argument or traditional argument (Lunsford, 1979); (2) Rogerian argument is not true to the essence of Rogers's theories, and thus does not deserve the name of "Rogerian" (Mader, 1980; Ede, 1984); (3) Rogerian rhetoric's requirement of empathetic listening and promotion of neutral language will further women's self-effacement (Lassner, 1990).

\section{A. Critique on Lack of Essential Difference from Aristotelian Rhetoric}

According to Lunsford's interpretation, the three key strategies of Rogerian rhetoric all have counterparts in Aristotelian rhetoric. The first point in Rogerian rhetoric is to convey to the reader that his ideas have been understood. This tenet, Lunsford (1979) believed, "is related closely to Aristotle's insistence that the rhetor must be able to argue on both sides of the question--indeed, must fully comprehend both sides of the question. To do so implied a full understanding, then, of the other person's positions and views" (p. 148). The second Rogerian strategy is to identify those aspects of the reader's position that are valid. This tenet, too, can be found in Aristotle, in his concept of the enthymeme (p. 149). The third strategy of Rogerian rhetoric is to induce the reader to believe that he and the writer have similar moral characteristics and goals. Lunsford (1979) demonstrated that "Aristotle's entire discussion of friendship and those factors which we love or like in other people continually stresses the importance of 'inducing similarities' "(p. 149).

Though much of what Lunsford has argued sounds convincing, a careful examination of the nature of Rogerian and traditional rhetoric supports, instead, the conclusion that the similarities are superficial while the differences are substantial. In his 1980 paper, Mader convincingly demonstrated why Rogerian rhetoric was not simply a "variation" on classic Aristotelian themes but fundamentally different. For example, in Rogerian rhetoric the writer must convince the reader that his position has been carefully considered and genuinely understood. For Aristotle, however, the reason to understand the other side is to know how best to refute it. As Mader (1980) said: "Aristotle's approach to 'understanding', therefore, is that of a partisan anxious to achieve victory." Hence the purpose of threat-reduction of the audience is exactly opposite to Aristotle's usage of the enthymeme, which is "to choose premises acceptable to the audience that make the speaker's conclusions valid" (p. 317).

\section{B. Critique on Unfaithfulness to Rogers' Theory}

Another main critique is that Rogerian rhetoric is not fully consistent with its source, Rogers' client-centered therapy. According to Ede, Rogerian rhetoric is not true to the three cornerstone conditions that Rogers requires a therapist to meet in order to truly carry out a patient-centered approach: congruence, or genuineness; unconditional positive acceptance or positive regard; and real empathetic listening. Ede (1984) stated her concern that "congruence requires one to abandon all efforts at interpretation, evaluation, or guidance" (p. 45). She also pointed out that Rogers' unconditional acceptance of any outcome, any direction of the therapeutic process had "little in common with Rogerian argument, which is clearly directed toward a goal, one established by the writer" (p. 45). Moreover, a therapist becomes immersed in the worldview of the client by making multiple attempts at understanding; it's difficult to translate this principle into writing, according to Ede, because the Rogerian writer much phrase the reader's accurately the first time (p. 45).

It is clear that Roger's theories were modified by Young, Becker and Pike when they proposed a new rhetorical approach. However, writers who embrace the Rogerian approach learn to use Rogers's attitude while following the Rogerian strategies, which, we believe, is true to the spirit of the Rogers despite limitations such as those posed by the inability to have constant feedback from the reader. The writer's sincere efforts to understand the reader is similar to Rogers' demand for genuineness, if not identical. Although writers of Rogerian rhetoric maintain the goal of problem-solving or mutual cooperation, they start the whole writing process with the positive goal of finding a win-win solution instead of prevailing over the other, even at the risk of being persuaded to the opponent's view, which is very close to unconditional positive regard. For the demand of empathetic listening, Rogerian writers strive diligently to gain a clear and comprehensive understanding, one that is close to what the reader believes. In particular, the Rogerian writer's efforts to explore contexts in which the reader's viewpoints are valid should be taken as sincere attempts to gain "complete immersion," even if they are aware of the impossibility of it. In conclusion, as Hairston pointed out in 1976: "we can use Rogers' approach to rhetoric in composition because the attitude is transferable, and it is the attitude that is the unique and crucial element" (p. 376). The Rogerian writer's painstaking efforts to meet the demands set up by Rogers clearly demonstrate that the attitude proposed by Rogers has been transferred to writing.

\section{Feminists'Main Critique}

A deeper criticism is from a feminist's perspective. Some of the feminist critiques indeed challenge the assumptions of the proponents and deserve our careful study.

1. Women Writers' Sense of Self-effacement

Lassner's paper "Feminist Reponse to Rogerian Argument" remains the most powerful and influential critique. Lassner's first main critique involves the "self-effacement" of women writers when the Rogerian approach is adopted. 
Lassner (1991) stated that "No writing can be empathetic unless it considers the reader's ideological assumptions as 'a politics of interpretation that is determined by race ....and gender' " (p. 222). Since Rogerian argument does not take gender into consideration in the process of being empathetic to the audience's perspectives, the empathy becomes a double bind on women, making them more "isolated" and "powerless." As Lamb (1991) pointed out, women have traditionally been the care-givers, the ones who work to understand others, even at their own expense (p. 17). Therefore, the requirement of empathy for the opponent as an essential part of an argument is "self-effacing" for women. To women writers, if Rogerian approach is adopted, it is further "feminine subservience."

2. Neutral Language Issue

The second major critique of Lassner centered on the "neutral language issue," which was explained by Brent (1996) as follows: "the detached, unemotional tone recommended by standard Rogerian rhetoric goes against the grain of most women's preferred ways of knowing" (p. 87). Lassner (1990) analyzed one of her student's responses to the detached approach and found that "to detach her emotion means, in effect, to deny her sense of herself" (p. 226). Even if women writers strive to be neutral, the emotion will still "crop up" in the empathetic restatement of the opponent's view, because it is too strong to be fully suppressed.

3. Responses to the Critique

To deal with the first problem of self effacement, we should keep in mind the difference between Rogers' psychotherapy and Rogerian rhetoric. In the patient-centered psychotherapeutic process, a therapist achieves full understanding of the patient's views by constant restatement without preset judgment. However, in Rogerian rhetoric, a rhetor not only accepts another's views without judgment, but also has the chance to delineate a different perspective. By doing so, women writers have an opportunity to present their own views and explain why they are different from the opposing ideas that they have summarized fairly (Brent, 1996, p. 87). Therefore, the requirement of empathetic understanding provides a rhetor with a chance to demonstrate full comprehension of the reader, which reinforces his or her understanding of the issue under discussion and wins the trust of the reader who will more likely be open to the rhetor's viewpoint.

To deal with the second problem, Brent (1996) suggested "'neutral' language must be valued not as a pure good in itself, but in a "dialectical relationship" with language that expresses emotion (p. 88). Although hostility should be avoided at all costs, this does not mean that a flat and totally detached tone should prevail; rather, writers are encouraged to avoid emotionally charged language when pure description fulfills the writer's purpose. Women writers are not, therefore, deprived of emotional language when writing Rogerian argument, but are advised, like any Rogerian writer, to avoid heavily-loaded language for an objective, but still forceful and effective statement.

\section{THE TEACHING OF ROGERIAN RHETORIC IN THE US AND ITS IMPLICATIONS FOR ENGLISH LEARNERS IN EAST ASIAN COUNTRIES}

Since Young, Becker and Pike articulated the Rogerian rhetoric in 1970, there have been many textbooks on written argument that introduce the basic theory or explain how Rogerian rhetoric can be taught to enrich the teaching of argument (Lamm \& Everett, 2007; Mauk \& Metz, 2006; Miller, 2007; Ramage \& Bean, 1998; White \& Billings, 2008; Wood, 1995). A number of published papers on Rogerian rhetoric are either fully devoted to the study of the pedagogy or incorporate teaching of Rogerian rhetoric as an important part (Hairston, 1982; Teich, 1992). For years writing teachers have been integrating Rogerian rhetoric into instruction in argument writing, especially at a time when American society seems to be becoming more polarized and adversarial, demonstrating the urgency for problem-solving instead of bickering with each other and sticking to the pro-con stereotype, leaving urgent questions unsolved for a long time (Tannen, 1998). This shows the enduring legacy of the approach, since it is being included as a viable option for American students who are learning argument writing. In some cases, theorists have used different terms to present essentially Rogerian rhetorical principles, such as "cooperative argumentation" (Makau \& Marty, 2001) or "conciliatory argument" (Kroll, 2005). Most argumentation textbooks, however, provide only brief introductions to the basic theory and structure, without sufficient discussion. Articles with practical advice on teaching Rogerian rhetoric are fewer in number but provide clearer suggestions for teachers. For example, Brent proposed systematic teaching methods, and Bator summarized the strategies that students need in order to deal with difficult phases; both of these articles contain advice that can be of great assistance to writing teachers who intend to incorporate a Rogerian approach in their classes.

\section{A. Doug Brent's General Suggestions}

In 1996, Brent discussed his teaching experience in an essay published in the book "Rogerian Rhetoric: Collaborative Rhetoric and Oral and Written Communication." In this essay, Brent suggested that in order to write Rogerian arguments, students need to learn how to "imagine with empathy." This goes beyond listing the audience's characteristics or opinions (traditionally called "audience analysis); instead students are asked to imagine the "entire worldview that allows those arguments to exist" and urged to "read with empathy." This process entails much more than a traditional procedure of researching the facts and statistics, because the writer must also explore the contexts in which the opponent's arguments are valid. To enable the empathetic view to "sink in," Brent proposes to teach in the following way:

(1) Pick a topic from a list of controversial issues and ask students to identify a side; 
(2) Call on a volunteer from each side to carry out a Rogerian type of discussion;

(3) If students fall into traditional debate mindset, and usually it does happen, invite students to distinguish Rogerian rhetoric from traditional rhetoric;

(4) Continue the exchange of restatements;

(5) Transcribe one's arguments into written form;

(6) Pair students and let them exchange papers and write summaries of the other paper;

(7) Correct one's statement according to the corresponding reader's response.

When students are capable of reflecting the views of the members of the audience who are present, students are led to write responses to newspaper articles using the Rogerian strategy, or more importantly adopting a Rogerian attitude.

\section{B. Paul Bator's Strategies to Deal with Difficult Phases}

Paul Bator studied students' responses to learning and applying a Rogerian approach and provided us with suggestions on how to assist students with difficult phases and help them use attentive language. To deal with the difficulty of restating the audience's position objectively, Bator suggested (1992) that students write with "two pens", which means to use separate paper for different views or use different files for opposing perspectives. Another method is to involve students in organized activities, in which students do role-playing of the audience and speaker positions, and then write a response from a primary audience perspective. Yet another approach is to encourage students to interview representative figures of the opposing position.

\section{Implication for Teaching in East Asian Countries}

Strategies suggested by Brent for enabling the empathetic view to sink in and Bator's on overcoming difficult phases, bring to light the main barrier to implementing Rogerian rhetoric in American institutions: the challenge of changing students' mindsets from striking for victory to listening with empathy. American college students have come of age in an "argument culture" (Tannen, 1998), an atmosphere that has taught them that arguing is a "win-lose" contest in which the major goal is to be a winner. Thus they have learned to argue by making a controversial claim, anticipating objections, attacking alternative positions, and arguing forcefully that others should accept their claim. They have learned this in school, but in fact most of them have already internalized this view of arguing from the broader culture. Some American teachers and scholars have become concerned about the corrosive effects of the "argument culture" and the limitations of teaching only a thesis-refutation-support pattern of arguing. Some of these educators have been drawn to Rogerian rhetoric, and for them the pedagogical problem is how to help students, who have been acculturated to the norm of adversarialism, learn to use different patterns of argument. At an even deeper level, the issue is how to encourage students to embrace the spirit of Rogerian rhetoric, which is rooted in the desire to understand another's point of view, as empathetically as possible, rather than to defeat an adversary. Thus the task is partly linguistic/rhetorical and partly ethical/attitudinal.

For East Asian students learning English, the situation is different. The cultural norms seem to be opposite: for East Asian students, raised in a heavily Confucianism influenced culture (Sung, 2007, p. 3), the norm is deference and accommodation. "Thus, extreme action such as hyperbole and polarized rhetoric are not favored in Confucius' doctrines and principles" (Cho, 1999, p. 55). Instead, the idea of approaching an argument by paying attention to the other person's position and looking for points of connection is familiar to East Asian students. Hence the spirit of Rogerian argument seems compatible with the East Asian norm for interacting with others. If one asks East Asian students to write according to a Rogerian pattern, there is a good chance that the pattern will seem familiar and less alienating than an argument with a strong thesis followed by refutation. Because the pattern is "comfortable," there is an opportunity to work on structures and linguistic matters. Nevertheless, even though East Asian students may not need attitudinal and ethical training, the exercises associated with Rogerian perspective taking are still good for developing language and rhetorical skills.

For those East Asian writing teachers who are interested in this approach, the following sequence of activities (based on Brent, 1996) might provide a way to introduce Rogerian argument. Students should select a controversial topic about which there are at least two opposing views - ideally a topic about which students have well-formed opinions. Students with similar opinions could work together to discuss why the topic is important and how it should be addressed, from their point of view. For East Asian students, this first step of clarifying their own position is crucial, even though they will not assert it in the first part of a Rogerian argument.

The second step is for students with opposing viewpoints to work in pairs, explaining their positions to one another. The task is to understand the other person's view so well that each can report the other's position to his or her satisfaction. This can be done orally (or, for more advanced students, in writing). The goal is to produce an accurate and fair-minded summary of the opposing view. From this activity, students learn the most important component of a Rogerian argument, which involves articulating the opposing viewpoint in neutral language.

The next step is to explore, in pairs, which elements in the opposing view one can affirm as valid in certain contexts. Even though positions differ, there are often deeper concerns or common values that opponents share. If so, this affirmation of elements of the opposing view provides a transition into the statement of the writer's viewpoint, which is the next part of a Rogerian argument. At this point, the assignment is to produce a written version of the writer's position and the arguments that support it, but in language that explains the position, rather than asserting it, and that 
refrains from refuting or critiquing the opposing view.

For the final essay, students will combine the various components: an introduction that focuses on a controversial issue or problem; a review of the opposing position, presented fairly and without rebuttal; and a statement about shared concerns and a transition into an explanation of the writer's different viewpoint. The last step is to add a conclusion that focuses, in more detail, both on those elements of the opposing viewpoint that the writer can support and also those aspects of the opposing view that would benefit from accepting some of the writer's suggestions. In this kind of a conclusion, differences are not fully resolved; nonetheless, the stage is set for understanding, cooperation, and further discussion. To complete this full argument, students should understand the overall structure and study a sample essay that illustrates it.

Following completion of a Rogerian argument, it might be instructive for students to contrast it with a more assertive argument on the same topic. The instructor could provide such a contrastive example, or, if teaching two classes at the same time, perhaps one group could work first on the traditional pattern and the other on a Rogerian argument, so that they could compare the resulting essays in terms of structure, diction, and tone. Each group would then proceed to write the contrasting type of argument, since facility at writing in both styles is ultimately beneficial. The goal is for students to choose an approach to argument that is appropriate for the audience and occasion.

In the process of completing the suggested activities, students will practice a variety of language skills, including brainstorming for supporting arguments, listening to an opposing viewpoint and summarizing it accurately, soliciting and transcribing comments from a listener, revising on the basis of readers' responses, and so forth. Therefore, the process of learning to argue in a Rogerian style will improve East Asian students' language skills, enhance their writing ability in general, and broaden their options for writing arguments.

Most importantly, Rogerian rhetoric teaches students the most fundamental lesson of rhetorical training: that choices must be appropriate to situations and audiences. When East Asian students are taught only the adversarial (thesis-support-refutation) strategy for arguing in English, they tend to exaggerate, over-generalize, and miss the subtleties of the approach. The result is ironic: East Asian students, who should tend toward deferential rhetoric, become even more aggressive and adversarial than most Americans find desirable, when they are encouraged to argue in the "American" manner. In a sense, they "over-learn" the distinctive features of American rhetoric. Therefore, introducing Rogerian rhetoric as an option, perhaps along with thesis-support-refutation patterns, might steer East Asian students away from inflexibility and rhetorical infelicities.

\section{REFERENCES}

[1] Bator, P. (1980). Aristotelian and Rogerian Rhetoric. College Composition and Communication, 31: 427-32.

[2] Bator, P. (1992). Carl Rogers and the Teaching of Rhetoric and Composition. In Teich, N. (eds.), Rogerian Perspectives: Collaborative Rhetoric for Oral and Written Communication (pp. 219-235). Norwood, NJ:Ablex.

[3] Brent, D. (1991). Young, Becker and Pike's "Rogerian" Rhetoric: A Twenty-Year Reassessment. College English, 53: $452-466$.

[4] Brent, D. (1996). Rogerian Rhetoric: Ethical Growth Through Alternative Forms of Argumentation. In Emmel, B., Resch, P. \& Tenney, D. (eds.), Argument Revisited; Argument Redefined (73-94). Thousand Oaks, CA: Sage Publications.

[5] Cho, J.H. (1999). A Study of Contrastive Rhetoric Between East Asian and North American Cultures as Demonstrated Through Student Expository Essays from Korea and United States. Retrieved from ProQuest Digital Dissertations.UMI Number 9930006

[6] Coe, R. M. (1992). Classical and Rogerian Persuasion: An Archoeological/Ecological Explication. In Nathaniel T. (eds.), Rogerian Perspectives: Collaborative Rhetoric for Oral and Written Communication (pp. 83-108). Norwood, NJ:Ablex.

[7] Corder, J. (1985). Argument as Emergence, Rhetoric as Love. Rhetoric Review, 4:16-32.

[8] Ede, L. S. (1984). Is Rogerian Really Rogerian? Rhetoric Review, 3:40-48.

[9] Hairston, M. 1976. Carl Rogers' Alternative to Traditional Rhetoric. College Composition and Communication, 27:373-377.

[10] Hairston, M. (1982). Using Carl Rogers' Communication Theories in the Composition Classroom. Rhetoric Review, 1:50-55.

[11] Kroll, B. M. (2005). Arguing Differently. Pedagogy. 5:37-60.

[12] Lamm, R. \& Everett, J. (2007). Dynamic Argument. Boston, MA: Houghton Mifflin.

[13] Lassner, P. (1990). Feminist Responses to Rogerian Argument. Rhetoric Review, 8: 220-31.

[14] Lunsford, A. A. (1979). Aristotelian vs. Rogerian Argument: A Reassessment. College Composition and Communication, 30: $146-51$.

[15] Mader, D. C. (1980). What Are They Doing to Carl Rogers? Et Cetera, 37:314-320.

[16] Makau, J. M. \& Marty, D. L. (2001). Cooperative Argumentation. Prospect Heights, IL: Waveland.

[17] Mauk, J. \& Metz, J. (2006). Inventing Arguments. Boston, MA: Thompson Higher Education.

[18] Miller, R. K. (2007). The Informed Argument. (7th ed.). Boston, MA: Thompson Higher Education.

[19] Ramage, J. D., \& Bean. J. C. (1998). Writing Argument (4th ed.). Boston: Allyn and Bacon.

[20] Rogers, C. R. (1970). Communication: Its Blocking and Facilitation. In Young, R. E., Becker, A. L., \& Pike, K. L. (eds.) Rhetoric: Discovery and Change, (pp. 284-289). New York: Harcourt.

[21] Sun, Y. H. (2007). The Influence of Culture on Parenting Practices of East Asian Families and the Impact on Emotional Intelligence of Older Adolescents. Retrieved from ProQuest Digital Dissertations.UMI Number 3258356.

[22] Tannen, D. (1998). The Argument Culture. New York: Ballantine.

[23] Teich, N. (1992). Rogerian Perspectives: Collaborative Rhetoric for Oral and Written Communication. Northwood, NJ: Alex.

[24] Thorne. S. F. (2003). Rogerian Rhetoric as Negotiation: Does Its Dependence on Game Theory Pose Ethical Problems? 2003, Winter. Http://reconstrcution.eserver.org: 
[25] White, F. D. \& Billings, S. J. (2008). The Well-Crafted Argument. (3rd ed.). Boston: Houghton Mifflin.

[26] Wood, N. V. (1995). Perspectives on Argument. Englewood Cliffs, NJ: Prentice-Hall.

[27] Young, R. E., Becker, A. L., \& Pike, K. L. (1970). Rhetoric: Discovery and Change. New York: Harcourt.

Yanling Guo was born in Jilin, China in 1975. She received her M.A. degree in linguistics from Northeast Normal University, China in 2002.

She is currently a lecturer in the School of Humanities, Beijing University of Posts and Telecommunications, Beijing, China. She has published eight papers and edited several books. Her major research interest is in English language teaching approaches.

Barry M. Kroll received his Ph.D in literature from University of Michigan, in 1997.

$\mathrm{He}$ is a Robert Rodale Professor of Writing. He has published many papers and books about teaching of writing. His major research interest is in writing methodology. 